\title{
Person Re-Identification Using Deep Learning Approach: A Survey
}

\author{
R. Mallika Pandeeswari ${ }^{1}$, S. Shargunam ${ }^{2}$ and G. Rajakumar ${ }^{3}$ \\ ${ }^{1,2 \& 3}$ Department of Electronics and Communication Engineering, \\ Francis Xavier Engineering College, Tamil Nadu, India \\ E-mail: mallikapandeeswari@francisxavier.ac.in, shargunamguna@gmail.com,gmanly12@gmail.com
}

\begin{abstract}
Person Re-identification proof crosswise over various observation cameras with disjoint fields of view has turned out to be one of the most intriguing and testing subjects in the zone of wise video observation. Albeit a few techniques have been created, what's more, proposed, certain confinements and uncertain issues remain. In the majority of the current Re-identification proof approaches, highlight vectors are separated from sectioned still pictures or video outlines. Distinctive similitude or disparity measures have been applied to these vectors. A few strategies have utilized basic consistent measurements, while others have used models to acquire streamlined measurements. Some have made models dependent on neighborhood shading or surface data, and others have constructed models dependent on the stride of individuals. When all is said in done, the primary goal of every one of these methodologies is to accomplish a higher-exactness rate and lower computational costs.

Keywords: Re-Ranking, Deep Learning, Machine Learning, Distance Measurement Similarity, Hash Function
\end{abstract}

\section{INTRODUCTION}

The Process of Matching a Person of interest among the images obtained from many cameras by assigning an ID to the different images is Person Re-Identification. Obtaining Persons from large collection from information sources. To track a Person to know their trajectory for surveillance as used in the forensic and Security application. Reidentification of people will have some serious consequences once, for instance, non-public healthdata is recovered that would cause discrimination, embarrassment, or maybe identity theft. Challenges Like over-exposure of lighting, occlusion, illumination is to be faced.

Fig. 1 shows a group of sample pictures for the subject below varied conditions, the images are taken from two surveillance camera sat various times of the day. The picture depicts subject intrinsic variations and changes in viewpoint, pose scale, and illumination conditions. One of the foremost tough challenges facing person reidentification methodology arises when the majority of clothing worn tends to be non-discriminative. Attributes-based strategies attempt to solve this drawback by incorporating attributes.
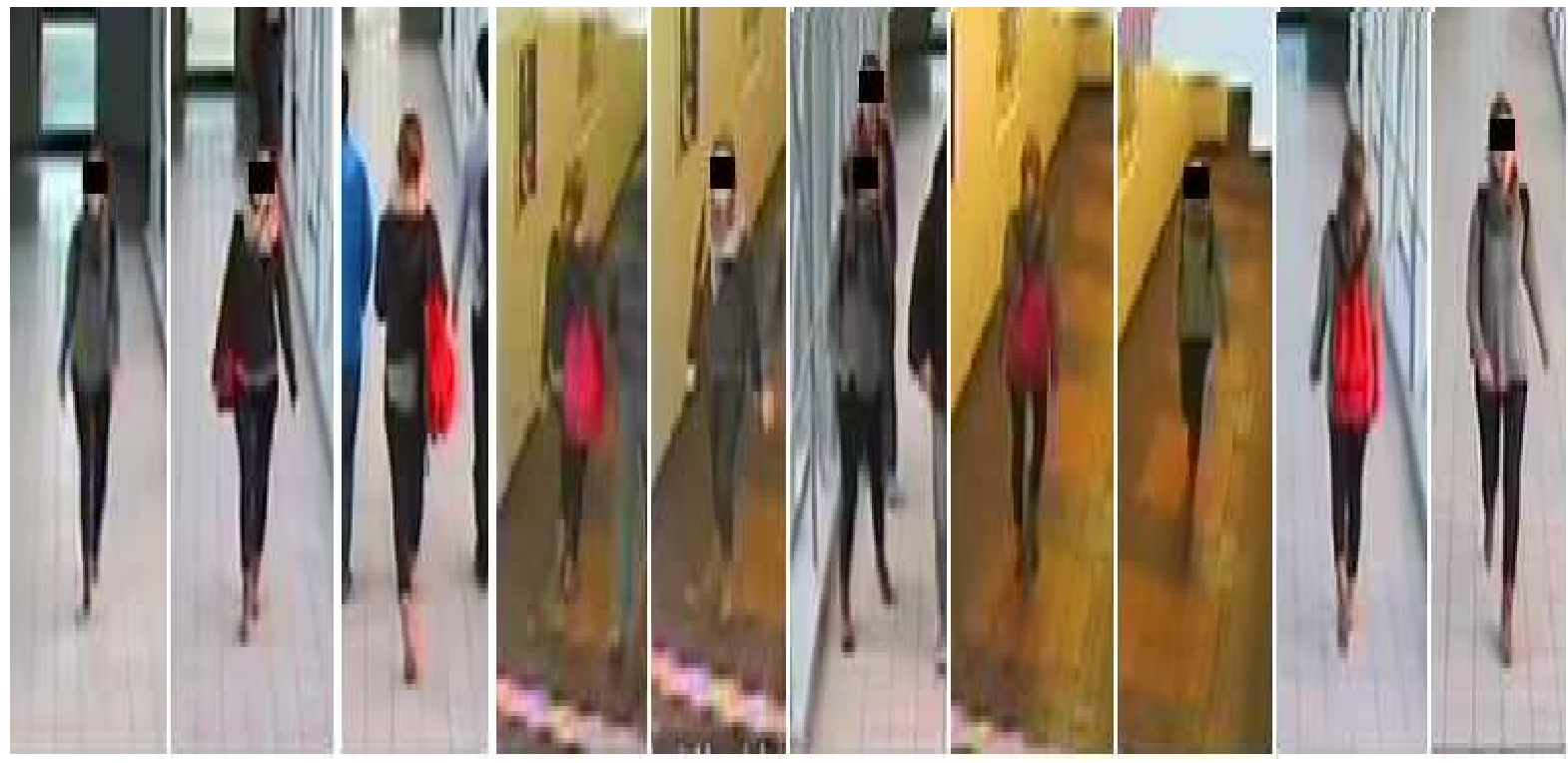

Fig. 1 Person re-identifcation challenges

'Jeans', 'male' and 'skirt' area unit all samples of linguistics attributes. Linguistics attributes area unit mid-level options learned from a larger dataset a priority. They provide an advantage when a single image is used to describe the person. In a model to mix linguistics attributes with lowlevel options area unit planned and shown to enhance the performance of person re-identification strategies. 


\section{RELATED WORKS}

This Paper proposed [1] Challenging issues and an overview of the current research in the problem of person re-identification. Two types of Re-Id tasks have been considered, closed set Re-Id and open set Re-Id. Open Set Re-Id is tracking images across multiple cameras coexisting in a time from a subset of the gallery. Open Set ReId is Many to Many Matching whereas a Single probe is presented and Gallery is fixed. Close-set Re-Id is too many matching. In the Non-Contextual Method, Visual Descriptors are used to characterize the person's appearance. In Descriptor Learning Method, presentation of Human silhouette as shape labels and appearance descriptors. Many Issues have been reported and hierarchical models are promoted to ease the issues.

This Paper described [2] image classification and image retrieval. Reviews are based on handcrafted and deeply learned systems. In the detection/tracking method bounding boxes are used to overlap similar images. In the gallery set, misalignment and misdetection are monitored by the threshold values. Re-Ranking in the Re-Id is given with an initial rank list with images ranked with top and low ranks. The detection error was corrected by the fine search for the Bounding Box. From the research and application views, reid in the very large gallery is marked as the critical direction in the future, and attempts to improve the accuracy and the efficiency issues are foretold. The inverted index-based and hashing-based solutions methods have been used to approximate nearest neighbor searches. The hash function was learned end to end by a deep learning network to output binary vector in the image classification dataset.

This paper modelled [3] their paper with the set of areas of appearance of people the spatial location of cameras, trajectories of people in non-observed regions in the field of view of the cameras. Selective patches are applied to the upper body of the persons in the crowded scenes where frequent occlusions are observed. Cumulative matching characteristics curve results from the best performance by providing people movement in the non-observed regions along with appearance method. An approach to re-id persons in the crowd was proposed based on regions of interest. As a Future Work, a study on how different appearance features affect the performance with the larger camera network and a dataset with people appearing only in a camera of creating a possibility to have false-positive reidentification.

This paper in a gist [4] can be stated that similar images are pulled whereas dissimilar ranked images are pushed away. That is true match images should not only be similar to strongly similar images of the probe but also be dissimilar to strongly dissimilar images in the gallery. With the Multi view verification model, a ranking aggregation algorithm was proposed with the view to improve the similarities and dissimilarities direction. The ranking aggregation method was based on conductive similarity and insulates dissimilarity. In the similarity ranking aggregation, an intersection set of top results with strongly similar images are treated as new probes to requery and are named as backward requery are enhanced by conducting cross-view based backward requery.

A refined ranking list was generated with graph-based weighted re-ranking. In Dissimilarities ranking aggregation strongly dissimilar images are obtained from the union set of bottom-k results and these are treated as new probes to requery their frequency in the ranking order. Future works proposed are to solve two problems based on the dataset size producing high computational cost and many not-sosimilar items regarding as similar images. To reduce the computational cost small subset was to be filtered out for improving ranking strategy.

This paper focuses [5] on traditional Gaussian features and deep learning of semantic features which are both used for discriminative of overall features. Image feature extraction was done to map the object in the image into a vector. Feature extraction was based on the color, texture gradient semantic. Distance Measurement similarity of two images was matched in the image library. Person feature descriptor Gaussian of Gaussian feature was extracted with their mean and normalization. Gaussian of Gaussian and convolutional neural network is fed into identification and verification network. Branch of Convolutional neural network, Gaussian of Gaussian feature are given as input data to predict person re-id instead of using the output to calculate similarities. Branch of Gaussian of Gaussian feature is combined with $\mathrm{CNN}$ which gives more information about the color, texture of images. GOG and CNN, mean, and normalization are removed. Future work was to explore the robust feature extraction method based on the fusion model.

This paper [6] describes the Multi-task learning Network comprised of pedestrian identity tasks and the attribute task, for person re-id with classification, verification, and Identification. The attribute task was to obtain the similarity in the images within the gallery setting, where attributes are divided into parts to ensure within part consistency along with Euclidean distance to find the distance in similarities between the images. The ranking was done to pick the closely related images. These images are made as to the image pool where re-ranking was done. The final ranking list was obtained by arranging the images in ascending order and was concluded by accomplishing the task of person reid using attribute labelling.

In this paper [7], the existing multi-shot works normally total single example include by processing the normal highlights of utilizing time arrangement model. The Multipicture Joint Re-positioning structure with an updateable Image Pool was proposed to give an alternate methodology. Euclidean distances of probe images and gallery images are calculated to sort in ascending order to form a ranking list. 
To begin with, the term 'Picture Pool' was characterized to store picture tests for each person on foot. Distance metrics were used to emphasizing inter-person distance and to depromote intra-person distance. Next, the refreshing standards of Image Pool have been characterized to advance representativeness. Second, we introductory positioning arrangements of each example in Image Pool are registered and proposed the 'Various picture Joint Re-positioning' calculation to total introductory positioning records. The rank score of incomplete components of introductory positioning records was figured. Future framework to further improve as a more real-world test.

This paper [8] proposed a new method for person reidentification by considering the pose of the person as a primary factor with multiple features extracted from important portions. For more robust matching and identification angle-based pose priority was applied. This method helps in reducing the number of images that are redundant in the training phase and the number of the matching process in the test phase. Under the illumination, viewpoint, occlusion variations, the strength of the proposed method was calibrated. The higher recognition rate for the issue of the identification process is obtained from the proposed approach. In future work, a new efficient feature and robust feature extraction method can be proposed for reidentification.

This paper [9] tackled the challenging problem of multi-shot person re-identification with the convolutional neural network. Two paradigms embedding space and instance space paradigms are developed to reformulate the person reidentification problem as a multi-instance variation problem extracted by a neural network with part-based features. Forward propagation on the dataset was performed and the output loss was computed. The gradients were then computed by applying back propagation.

True positives, false positives, and false negatives were labeled in the gallery set. A predicted similarity probability between the samples from the discriminative to noninformative (imposters) is marked. A specific bag level loss function was a device to incorporate the characteristics of the multi-instance problems for each paradigm. From the experimental study, the instance paradigm has been verified to be the most efficient approach as well as having a better power to determine the discriminative and non-informative instance pairs for better verification performance in the multi-instance part-based feature convolutional neural network re-formulation in the task of person reidentification.

TABLE I COMPARISON OF TECHNIQUES USED IN DEEP LEARNING

\begin{tabular}{|c|c|c|c|}
\hline Sl. No. & Author & Technique Used & Objective \\
\hline 1 & $\begin{array}{l}\text { Apurva Bedagkar- Gala } \\
\text { et al., }\end{array}$ & Descriptor Learning Method & $\begin{array}{l}\text { Presentation of Human silhouette as shape labels } \\
\text { And appearance descriptors. }\end{array}$ \\
\hline 2 & Liang Zheng et al., & Hash function Method & $\begin{array}{l}\text { The hash function was learned end to end by a deep } \\
\text { learning network to output binary vector in the } \\
\text { image classification dataset. }\end{array}$ \\
\hline 3 & Kaiyang Zhou et al., & $\begin{array}{l}\text { convolutional neural networks } \\
\text { Method }\end{array}$ & $\begin{array}{l}\text { emphasizing common local pattern by using } \\
\text { convolutional neural networks to obtain a relevant } \\
\text { descriptor }\end{array}$ \\
\hline 4 & Dapeng Chen et al., & Polynomial Feature & learning methods to establish spatial constraints \\
\hline 5 & Nanning Zheng et al., & Cumulative matching characteristics & $\begin{array}{l}\text { The appearance of people the spatial location of } \\
\text { cameras, trajectories of people in non-observed } \\
\text { regions in the field of view of the cameras. }\end{array}$ \\
\hline 6 & Zheng Wang et al., & $\begin{array}{l}\text { conductive similarity and insulative } \\
\text { dissimilarity }\end{array}$ & $\begin{array}{l}\text { Similar images are pulled whereas dissimilar ranked } \\
\text { images are pushed away. }\end{array}$ \\
\hline 7 & Yang Fu et al., & partial discriminative feature & $\begin{array}{l}\text { specific person parts, maximum pooling extracted } \\
\text { information and ignored those interfaces extract } \\
\text { similar clothing and background }\end{array}$ \\
\hline 8 & Nan Song et al., & Distance Measurement similarity & $\begin{array}{l}\text { Gaussian features and deep learning of semantic } \\
\text { features are both used for discriminative of overall } \\
\text { features. }\end{array}$ \\
\hline 9 & Hefei Ling et al., & Multi-task Learning & The task of person re-id using the attribute labeling. \\
\hline 10 & Yuan et al., & Re-Ranking Method & $\begin{array}{l}\text { Top candidates are re-ranked to improve the } \\
\text { accuracy of the ranking results. }\end{array}$ \\
\hline 11 & Liu et al., & Instance level recognition problem & To Match a specific person of interest in a network. \\
\hline 12 & Chen et al., & Outlier misalignment & To detect the outlier based on person misalignment. \\
\hline
\end{tabular}


This paper [10] proposed a robust re-ranking method based on an expanded k-reciprocal neighbour approach. A gallery image as the probe image was assumed likely to be the same person by the expanded k-reciprocal nearest neighbour.

The probe image was replaced with the expanded reciprocal nearest neighbour and the final distance was determined by the mean value of the corresponding neighbour set. This method was an automatic, unsupervised re-id problem. A simple direct rank list can be performed well with this method. The nearest neighbour is introduced to reduce the interference of mismatched pairs and the similarity between the probe and the gallery is discovered to compare distance in the re-ranking system. Feature extraction, metric learning, re-ranking are used to enhance the re-id model learning. The false positives in the ranking list are eliminated by the expanded k-reciprocal nearest neighbour. Hence this fully automatic unsupervised model works with known re-computation for any rank lists.

This Paper [11] described an instance-level recognition problem where a person re-id relying on the discriminative features capturing not only the different spatial scales but also incorporating an arbitrary combination of multiple scales. A novel deep re-id neural network was designed and was termed as Omni-Scale network (Osnet) to achieve a residual block composed of multiple convolutional streams which can detect features at a certain scale. A unified aggregation gate was used to dynamically fuse multi-scale features with input depended on channel-wise weights. Stacking the blocks layer-by-layer achieved the state of the art performance.

This paper [12] focuses on relief issues like partial discriminative feature representation. In the local region generation process poses, discriminative region, salient region based on the region of interest with deep feature Maps, crop deep feature map into predefined patches perfectly aligned. The negative effect caused by unexpected and unaligned causes for horizontal pyramid pooling. Multiple scale issue caused by an outlier of misalignment was observed. To enhance discriminative information of all specific person parts, maximum pooling extracted information and ignored those interfaces extract similar clothing and background. Column feature input into nonshape fully connected layers with the soft max predicts id of the input image is gained. The loss function was used to tackle person re-identification. It's feature representation learned by the classification model to maintain the global relation with identification.

\section{CONCLUSION}

This paper initially presents the customary techniques for person re-ID. Deep learning utilizes its Deep structure to gain includes consequently from a lot of information, staying away from the inadequacies of conventional manual component extraction techniques. The same number of issues exists, there are still some important research bearings later on. We need continually center around how to apply profound adapting better to make Person Reidentification proof applied to the genuine circumstance.

\section{REFERENCES}

[1] A. Bedagkar-Gala and S. K. Shah, "A survey of approaches and trends in person re-identification,” Image and vision computing, Vol. 32, No. 4, pp. 270-286, 2014.

[2] L. Zheng, Y. Yang and A. G. Hauptmann, "Person re-identification: Past, present, and future," arXiv preprint arXiv: 1610.02984, 2016.

[3] L. Wu, Y. Wang, X. Li and J. Gao, "What-and-where to match: Deep spatially multiplicative integration networks for person reidentification,” Pattern Recognition, Vol. 76, pp.727-738, 2018.

[4] D. Chen, Z. Yuan, B. Chen and N. Zheng, "Similarity learning with spatial constraints for person re-identification," In Proceedings of the IEEE conference on computer vision and pattern recognition, pp. 1268-1277, 2016.

[5] R. Mazzon, S. F. Tahir and A. Cavallaro, "Person re-identification in crowd," Pattern Recognition Letters, Vol. 33, No. 14, pp.1828-1837, 2012.

[6] M. Ye, C. Liang, Y. Yu, Z. Wang, Q. Leng, C. Xiao, J.Chen and R. $\mathrm{Hu}$, "Person re-identification via ranking aggregation of similarity pulling and dissimilarity pushing," IEEE Transactions on Multimedia, Vol. 18, No. 12, pp. 2553-2566, 2016.

[7] Y. Fu, Y. Wei, Y. Zhou, H. Shi, G. Huang, X. Wang, Z. Yao and T. Huang, "Horizontal pyramid matching for person re-identification," In Proceedings of the AAAI Conference on Artificial Intelligence, Vol. 33, No. 01, pp. 8295-8302, 2019.

[8] Y. Liu, N. Song and Y. Han, "Multi-cue fusion: Discriminative enhancing for person re-identification", Journal of Visual Communication and Image Representation, Vol. 58, pp. 46-52, 2019.

[9] Y. Lin, L. Zheng, Z. Zheng, Y. Wu, Z. Hu, C. Yan, and Y. Yan, "Improving person re-identification by attribute and identity learning."Pattern Recognition, Vol. 95, pp. 151-161, 2019.

[10] M. Yuan, D. Yin, J. Ding, Z. Zhou, C. Zhu, R. Zhang, and A. Wang, "A multi-image Joint Re-ranking framework with updateable Image Pool for person re-identification," Journal of Visual Communication and Image Representation, Vol. 59, pp. 527-536, 2019.

[11] X. Liu, S. Bi, X. Ma and J. Wang, "Multi-Instance Convolutional Neural Network for multi-shot person re-identification," Neuro computing, Vol. 337, pp. 303-314, 2019.

[12] Y. Chen, J. Yuan, Z. Li, Y. Wu, M. Nouioua and G. Xie, "Person reidentification based on re-ranking with expanded k-reciprocal nearest neighbors," Journal of Visual Communication and Image Representation, Vol. 58, pp. 486-494, 2019. 